\title{
Attitude Determination from Single-Antenna Carrier-Phase Measurements
}

\author{
Thomas B. Bahder \\ U. S. Army Research Laboratory \\ 2800 Powder Mill Road \\ Adelphi, Maryland, USA 20783-1197
}

(October 30, 2018)

\begin{abstract}
A model of carrier phase measurement (as carried out by a satellite navigation receiver) is formulated based on electromagnetic theory. The model shows that the phase of the open-circuit voltage induced in the receiver antenna with respect to a local oscillator (in the receiver) depends on the relative orientation of the receiving and transmitting antennas. The model shows that using a single receiving antenna, and making carrier phase measurements to seven satellites, the 3-axis attitude of a user platform (in addition to its position and time) can be computed relative to an initial point. This measurement model can also be used to create high-fidelity satellite signal simulators that take into account the effect of platform rotation as well as translation.
\end{abstract}

\section{INTRODUCTION}

Satellite systems such as the Global Positioning System (GPS) and the Global Navigation Satellite System (GLONASS) were originally designed to provide a user with position and time [1,2, 3]. In addition to position and time, orientation (with respect to some system of coordinates) or attitude information has numerous applications, such as to aircraft flying by instrument navigation, pointing communications antennas and satellites, unmanned air vehicles (UAV), and as a source of compass heading. In recent years, attitude (or orientation) information has been obtained from the GPS by using multiple, widely-separated antennas mounted on a (assumed) rigid platform [4. When the rigid platform translates and rotates, the path length changes between the transmitting satellite and each receiving antenna. Each satellite transmits a (modulated) continuous wave sinusoidal signal. The phase of the voltage induced in each receiving antenna changes with respect to the phase of a local oscillator in the receiver. A measurement of this effect is called a carrier phase measurement (or delta-range measurement) and is usually modeled [4 as the phase difference (or accumulated phase difference from some point in time) between the receiver's internal oscillator and the voltage induced on the given receiving antenna.

In current GPS receiver technology, this phase change is modeled as arising solely from the change in path length between transmitting satellite and receiver antenna [4]. This model is used in commercial GPS receivers to determine the current orientation or attitude of the rigid platform (on which the antennae are mounted) relative to the (platform) attitude at some initial point in time. The standard model of carrier phase measurement [4] completely neglects the physical nature of the electromagnetic field (i.e., that it is a vector field) and the nature of the detection process that occurs in a real antenna. The role of the antenna is to convert the vector electromagnetic field into a time-dependent voltage signal.

In recent work, Krall and Bahder [5,6] developed an improved physical model of the carrier phase measurement process. They have shown that using a single dipole antenna two angles of attitude can be obtained by tracking six satellites [5.6]. Their model of carrier phase measurement treats the electromagnetic field as a vector and includes orientation effects of the detection process of the receiving antenna. In particular, the model of Krall and Bahder [5] 6] shows that the phase of the open circuit voltage induced in the receiving antenna depends on the relative orientation of the receiving and transmitting antennas, which is a feature that is neglected by the standard model [ [I] of carrier phase measurement [7].

The equations presented by Krall and Bahder [5.60] are specific to a dipole receiving antenna and these equations cannot be simply extended to more realistic types of receiving antennas, such as those that are currently used in actual GPS receivers. Furthermore, the Krall and Bahder equations allow determination of only two angles of attitude, while many applications require knowledge of all three angles of attitude for a user platform.

In this paper, I generalize the work of Krall and Bahder. I show that, for a general type of antenna, all three angles of attitude can be computed from carrier phase measurements, i.e., when the receiving antenna has no cylindrical symmetry. I present a generalized model of the carrier phase measurement process based on the vector effective length of an arbitrary antenna. The vector effective length characterizes a receiving antenna in terms of a two-component complex vector field, $\mathbf{h}(\theta, \phi)$, on the surface of a unit sphere surrounding the antenna [8, 9, 10, 11]. The spherical polar angles $\theta$, and $\phi$ specify the direction from which the radiation is incident on the receiving antenna, as seen in the comoving frame of the receiving antenna. 
The improved model of carrier phase measurement developed below can be used to compute all three angles of platform attitude from single-antenna carrier phase measurements to seven satellites. Since the phase of the voltage induced in the receiving antenna depends on the relative orientation of the transmitting and receiving antennas, the attitude of the satellite antennas must be known to the user of the system. For the case of GPS, the satellite orientation is quite strict because the power in the main antenna beam is centered on the Earth 12. So the satellite antenna attitude is assumed known in the model presented below. However, for future high-accuracy attitude determination, the satellite system should have its navigation message enhanced so that it contains information on the orientation of each satellite antenna. As a result of this enhancement, the GPS would be a a navigation system that provides users with their position, time, and orientation (or attitude).

The single-antenna feature of the model presented below permits miniaturization of the attitude determination system over that of present systems, since multiple antennas are not needed. Consequently, this model is advantageous in applications that require miniaturization, such as pointing communications antennas, nano-satellites [13], micro air vehicles (MAV) [14], and wrist-mounted compass heading systems [15]. This model can also be used for developing a satellite signal simulator that faithfully models carrier phase measurements made by a platform that undergoes rotation (in addition to translation) such as a satellite, aircraft or missile.

Additionally, in the currently-used multiple-antenna attitude determination systems, higher accuracy of attitude is achieved with larger antenna separations (baselines). However, in systems with large baselines, alignment of the antennas, as well as change of antenna orientation due to flexing of the (assumed rigid) mounting base, are complications that can lead to significant phase errors. For this reason, a single-antenna attitude determination system may be preferable. Alternatively, it may be advantageous to use the model of carrier phase measurement presented below in multiple antenna systems so that the requirements of platform rigidity and antenna alignment can be relaxed.

The apparent phase center of a receiving antenna moves as a function of direction of the incident radiation from the satellite. The direction from which the signal arrives at the receiving antenna, as seen in the comoving frame of the receiving antenna, depends on the relative velocity of the transmitting and receiving antennas (aberration of star light effect). This effect was taken into account by Krall and Bahder to first order in $v / c \sim 10^{-5}$, where $v$ is the speed of the satellite antenna in the comoving frame of the receiving antenna, and $c$ is the speed of light. For typical satellite navigation applications, the speed of the receiver is small (compared to $c$ ) and this aberration effect amounts to approximately $10^{-5}$ radians of angle, and therefore can be neglected. In this work, I neglect this first order effect by dropping terms of first order in $v / c$. However, since the orientation effects (such as dependence of the phase of the voltage on direction of incident radiation and movement of the apparent phase center) are zeroth-order effects, they are included in the model below.

In this paper, the standard model of carrier phase measurement is reviewed in section II. Section III, contains a calculation of the open-circuit voltage induced in a receiving antenna due to the satellite signal. The carrier phase measurement model based on the open-circuit antenna voltage is formulated in section IV. Section V deals with application of the measurement model to attitude determination and navigation. Section IV presents a summary of the ideas involved in this work.

\section{STANDARD MODEL OF CARRIER PHASE MEASUREMENT}

The standard multiple-antenna technique of attitude determination relies on a simplified model of carrier phase measurements [4]. This standard technique makes use of differences between the phase of a local oscillator and the phase of the incoming satellite signal, at the positions of the (multiple) receiving antennas. In this technique, the satellite signal is essentially treated as a scalar wave of amplitude $E$, given by

$$
E=E_{o} e^{-j(k r-\omega t)}
$$

where $E_{o}$ is a real amplitude, $r=|\mathbf{r}-\mathbf{R}|$ is the distance from the satellite antenna at transmission point $\mathbf{R}$ to receiver antenna at reception point $\mathbf{r}$, the angular frequency $\omega=2 \pi f, f$ is the frequency of the carrier signal and $j=\sqrt{-1}$. The wave propagates with wave vector $k=N \omega / c=2 \pi N / \lambda$, at speed $c / N$, where $c$ is the speed of light and $N$ is the average index of refraction over the signal's path. In the simplified model of carrier phase measurements, the measured phase is taken to be the phase of the scalar field, seen by antenna $i$ at time $t$ at position $\mathbf{r}_{i}(t)$, due to a satellite $j$ at position $\mathbf{R}_{j}(t-\tau)$ :

$$
\phi_{i j}(t)=k\left|\mathbf{r}_{i}(t)-\mathbf{R}_{j}(t-\tau)\right|-\omega t
$$

where $\tau$ is the time of flight of the signal, implicitly defined by 


$$
\tau=\frac{\left|\mathbf{r}_{i}(t)-\mathbf{R}_{j}(t-\tau)\right|}{c}
$$

The satellite receiver has a local oscillator that is not perfect. The imperfection of the oscillator is represented by the fact that the oscillator does not keep time according to Earth-centered inertial (ECI) coordinate time or universal coordinated time (UTC), $t$, but according to its own clock that counts cycles 16] according to a time scale $t^{*}$, which is some function of, say, ECI time $t$. The oscillator will then have a running phase given by

$$
\phi_{\text {osc }}(t)=-\omega t^{*}
$$

where the time scale $t^{*}$ is not the same as the ECI (or UTC) time scale $t$, but there is a functional relation $t^{*}=t^{*}(t)$ between these two times. The change in phase of the local oscillator since some initial time or epoch, $t_{o}$, can then be represented by

$$
\Delta \phi_{\text {osc }}(t)=-\omega\left(t^{*}-t_{o}\right)
$$

The difference between the ECI coordinate time elapsed since time $t_{o}, t-t_{o}$, and the elapsed time as computed by counting oscillator cycles, $t^{*}-t_{o}$, is given by $\left(t-t_{o}\right)-\left(t^{*}-t_{o}\right)=t-t^{*} \equiv \Delta t^{*}$, where $\Delta t^{*}$ is the oscillator clock bias (error) that has accrued since the initial time $t_{o}$. So the time kept by the oscillator, $t^{*}$, and the coordinate time, $t$, are related by

$$
t^{*}=t-\Delta t^{*}
$$

In the simplified model of carrier phase measurement 何, the carrier phase measured by a receiver is taken to be the phase difference between the incoming wave (at the antenna phase center) and that of the local oscillator, given by

$$
\Delta \phi_{i j}(t)=\phi_{i j}(t)-\phi_{o s c}(t)=k\left|\mathbf{r}_{i}(t)-\mathbf{R}_{j}(t-\tau)\right|-\omega \Delta t^{*}+2 \pi m_{i j}
$$

where the integer $m_{i j}$ is needed since the actual phase measurement can only be done up to an integer number of cycles. For example, if the geometric range between receiver and satellite is increased by an integer number of wavelengths, the measured phase difference, as given in Eq. (耳), would be the same due to a different integer.

By taking the origin of coordinates near the user platform, and taking differences of the measured quantities in Eq. (7), the clock bias $\Delta t^{*}$ can be eliminated from the unknowns, leaving only geometric quantities, such as the baseline vectors between antennas, $\mathbf{r}_{i}-\mathbf{r}_{k}$, a unit vector to the satellite, $\mathbf{R}_{j} /\left|\mathbf{R}_{j}\right|$, and unknown integers. For example, for different antennae $i, k$ tracking the same satellite $j$, the difference of phases given by Eq. (7) is

$$
\begin{aligned}
\Delta \phi_{i j}(t)-\Delta \phi_{k j}(t) & =k\left(\left|\mathbf{r}_{i}-\mathbf{R}_{j}\right|-\left|\mathbf{r}_{k}-\mathbf{R}_{j}\right|\right)+2 \pi\left(m_{i j}-m_{k j}\right) \\
& \approx k\left\{\left(\mathbf{r}_{k}-\mathbf{r}_{i}\right) \cdot \frac{\mathbf{R}_{j}}{\left|\mathbf{R}_{j}\right|}\right\}+2 \pi\left(m_{i j}-m_{k j}\right)
\end{aligned}
$$

where $\left|\mathbf{r}_{i}\right|,\left|\mathbf{r}_{k}\right|<<\left|\mathbf{R}_{j}\right|$. In the simplest case, to determine the platform attitude, we need three antennae to track two satellites. Two unknown baseline vectors, say $\left(\mathbf{r}_{1}-\mathbf{r}_{2}\right)$ and $\left(\mathbf{r}_{2}-\mathbf{r}_{3}\right)$, uniquely determine the plane (attitude) of the platform. Each of these baseline vectors has three unknown components, so there are six unknowns. Therefore, tracking two satellites, $j=1,2$, with each of the three antennas, $i=1,2,3$, results in six measurements $\Delta \phi_{i j}(t)$. The six difference equations given by Eq. (9) can then be solved (up to integers) for the six vector components of the two baseline vectors, $\mathbf{r}_{k}-\mathbf{r}_{i}$, for say $i=1$ and $k=1,2$.

The standard technique [4] for attitude determination, described in Eq. (1)-(9), uses a measurement model of carrier phase given by Eq. (7), which essentially treats the electromagnetic field as a scalar field, and neglects its vector nature. The electromagnetic vector field carries additional orientation information that can be exploited to determine attitude of a user platform. Recently, it was shown that, using a short-dipole receiving antenna to track signals from six GPS satellites, two angles of attitude can be determined [6,5], i.e., the orientation of the antenna can be determined up to rotations about its axis. The method used to derive these results was limited to a short-dipole type of receiving antenna, and to a determination of only two of the three Euler angles of attitude [6.57.

In what follows, I show that using a single receiving antenna of arbitrary type, and tracking the carrier phase from seven satellites, the complete 3 -axis attitude (in addition to position and time) can be determined. The results here are based on a model of the open-circuit voltage induced in the receiving antenna. The model explicitly takes into account the vector nature of the electromagnetic field that is broadcast by the satellite. The model shows that the phase of the voltage induced in the receiving antenna contains an additional phase-shift $\delta_{s}(t)$, see Eq. (32), that depends on the relative orientation of the transmitter and receiver antennas. Consequently, given the position and orientation of the transmitting satellite antennae, the orientation (3-axis attitude) of the receiving antennae can be computed. 


\section{OPEN-CIRCUIT ANTENNA VOLTAGE}

The basis of the single-antenna method of attitude determination is the open-circuit voltage $V_{s}(t)$ induced in a receiving antenna [8,9,10,11] by the radiation field from satellite $s$ :

$$
V_{s}(t)=\mathbf{h}\left(\mathbf{n}_{s}\right) \cdot \mathbf{E}_{s}\left(\mathbf{R}_{o}, t\right)
$$

where $\mathbf{E}_{s}\left(\mathbf{R}_{o}, t\right)$ is the electric radiation field at the receiver antenna located at position $\mathbf{R}_{o}$ at reception time $t$ and $\mathbf{h}\left(\mathbf{n}_{s}\right)$ is the receiving antenna vector effective length (or effective height), which characterizes the antenna. The unit vector $\mathbf{n}_{s}$ specifies the direction of field propagation, as seen in the comoving frame of the receiving antenna. The origin of this comoving system of coordinates is centered at the receiving antenna, which is at point $O$. See Fig. 1 . In Eq. (10), both the electric field, $\mathbf{E}_{s}\left(\mathbf{R}_{o}, t\right)$, and the vector effective height, $\mathbf{h}\left(\mathbf{n}_{s}\right)$, are vectors in the comoving frame of reference of the receiver. The vector $\mathbf{n}_{s}$ is given in terms of the polar coordinate angles $\theta_{s}$ and $\phi_{s}$, in the receiving antenna's comoving frame:

$$
\mathbf{n}_{s}=-\left[\sin \theta_{s} \cos \phi_{s} \mathbf{a}_{1}+\sin \theta_{s} \sin \phi_{s} \mathbf{a}_{2}+\cos \theta_{s} \mathbf{a}_{3}\right]
$$

Here, $\mathbf{a}_{1}, \mathbf{a}_{2}, \mathbf{a}_{3}$ are the orthonormal unit vectors in the comoving system of coordinates that is centered at the receiving antenna. Neglecting relativistic aberration, this direction of propagation can be specified by a unit vector $\mathbf{n}_{s}$, pointing from the satellite to the receiver, in the ECI coordinate system [17].

The vector effective height, $\mathbf{h}\left(\mathbf{n}_{s}\right)$ in Eq. (10), is a two-dimensional complex vector field on the unit sphere surrounding the receiving antenna that describes the receiving properties of an arbitrary antenna. In general, the field $\mathbf{h}(\theta, \phi)$ is specified on the unit sphere by two complex functions, $h_{\theta}(\theta, \phi)$ and $h_{\phi}(\theta, \phi)$,

$$
\mathbf{h}(\mathbf{n})=h_{\theta}(\theta, \phi) \mathbf{a}_{\theta}+h_{\phi}(\theta, \phi) \mathbf{a}_{\phi}
$$

where $\theta$ and $\phi$ are the polar angles (coordinates on the unit sphere), and $\mathbf{a}_{\theta}$ and $\mathbf{a}_{\phi}$ are orthonormal basis vectors on this sphere. The origin of coordinates is co-located with the receiving antenna. In practice, the functions $h_{\theta}(\theta, \phi)$ and $h_{\phi}(\theta, \phi)$ must be found by computation, or, by experiment. In the case of experiment, these two functions are found by mapping the far-field pattern of the receiving antenna, when it is used in the transmission mode. Then, using the equation for the far-field [9, 11

$$
\mathbf{E}_{a}=E_{\theta}(\theta, \phi) \mathbf{a}_{\theta}+E_{\phi}(\theta, \phi) \mathbf{a}_{\phi}=-j \sqrt{\frac{\mu}{\epsilon}} \frac{k I_{i n}}{4 \pi r} \mathbf{h}(\theta, \phi) e^{-j(k r-\omega t)}
$$

where $I_{i n}$ is the current through the antenna, $\epsilon$ is the permittivity, $\mu$ is the permeability of the surrounding medium, and $r$ is the distance from the antenna, the components of the vector effective height, $h_{\theta}(\theta, \phi)$ and $h_{\phi}(\theta, \phi)$, can be determined. Note that this procedure is well-defined for any type of receiving antenna. For a discussion of measurements and theory of elliptically polarized fields, see Ref. [8, 9, 10,11] and the coordinated series of papers in Ref. [18, 19,20,21].

The electromagnetic field broadcast by the satellite, at the position of the receiver, is a far-field. In this far-field region, the general form of the electromagnetic field at receiver position $\mathbf{R}_{o}$ at time $t$ is given by the real part of

$$
\mathbf{E}\left(\mathbf{R}_{o}, t\right)=\left[\mathbf{u} E_{u}(\Theta, \Phi) e^{\psi_{u}(\Theta, \Phi)}+\mathbf{v} E_{v} e^{\psi_{v}(\Theta, \Phi)}\right] \frac{e^{-j k r}}{r} e^{j \omega t}
$$

where the vectors $\mathbf{u}$ and $\mathbf{v}$ are real orthogonal unit vectors that specify the polarization of the field and the range, $r=\left|\mathbf{R}_{o}-\mathbf{R}_{T}\right|$, is the distance between reception event at the receiver $\left(t, \mathbf{R}_{o}\right)$ and emmission event at the satellite $\left(t_{T}, \mathbf{R}_{T}\right)$. The quantities $E_{u}$ and $E_{v}$ are real amplitudes that depend on polar angles $\Theta$ and $\Phi$, measured from the origin at point $T$ at the satellite antenna. See Fig. 1. Each polarization can have an arbitrary phase $\psi_{u}$ and $\psi_{v}$. The field given by Eq. (14) is a general elliptically polarized electric field.

As an example of a specific case of Eq. (14), consider a GPS satellite, where the field near the central beam axis is a right circularly polarized field, given by

$$
\mathbf{E}\left(\mathbf{R}_{o}, t\right)=[\mathbf{u}-j \mathbf{v}] E(\Theta, \Phi) \frac{e^{-j k r}}{r} e^{j \omega t}
$$

where $E(\Theta, \Phi)$ is a real amplitude. If the field broadcast by the satellite is not a right circularly polarized field, then Eq. (15) still applies, however, the vectors $\mathbf{u}$ and $\mathbf{v}$ will have complex phases, $\mathbf{u}=\mathbf{u}_{o} e^{j \phi_{u}}$ and $\mathbf{u}=\mathbf{v}_{o} e^{j \phi_{v}}$, where $\mathbf{u}_{o}$ 
and $\mathbf{v}_{o}$ are real orthogonal unit vectors. Consequently, no generalization is lost by using Eq. (15) for the far-field of the satellite. This representation is used below.

Strictly, $\mathbf{E}\left(\mathbf{R}_{o}, t\right)$ in Eq. (14) is the electric field in the comoving frame of the satellite, while $\mathbf{E}_{s}\left(\mathbf{R}_{o}, t\right)$ in Eq. (10) is the field in the comoving frame of the receiver. The difference between these two fields is a quantity of first order in $v / c$, where $v$ is the speed of the satellite in the frame of reference of the receiver. In what follows, I drop these first order terms in $v / c$, taking $\mathbf{E}_{s}\left(\mathbf{R}_{o}, t\right) \approx \mathbf{E}\left(\mathbf{R}_{o}, t\right)$. Furthermore, the vectors $\mathbf{u}$ and $\mathbf{v}$ in Eq. (15) are taken at the retarded time $t-r_{s} / c$, which is the time at which the signal left the satellite.

Using the far-field of the satellite given by Eq. (15), the open-circuit voltage induced in the receiver antenna due to the electromagnetic field from satellite $s$, given in Eq. (10), can be written as (summation over repeated indices):

$$
V_{s}(t)=h_{i}\left(\mathbf{n}_{s}(t)\right) D_{i k}(t)\left[R_{1 k}\left(t-\frac{r_{s}}{c}\right)-j R_{2 k}\left(t-\frac{r_{s}}{c}\right)\right] E\left(\mathbf{n}_{s}\right) \frac{e^{-j k r}}{r} e^{j \omega t}
$$

Here, $\mathbf{n}_{s}(t)$ is the unit vector from satellite $s$ at position $\mathbf{R}_{s}\left(t-r_{s} / c\right)$ at emmision event time $t-r_{s} / c$, to the receiver at position $\mathbf{R}_{o}(t)$ at reception event time $t$, given by 17

$$
\mathbf{n}_{s}(t)=\frac{\mathbf{R}_{o}(t)-\mathbf{R}_{s}\left(t-\frac{r_{s}}{c}\right)}{\left|\mathbf{R}_{o}(t)-\mathbf{R}_{s}\left(t-\frac{r_{s}}{c}\right)\right|}
$$

where the positions of the receiver $\mathbf{R}_{o}(t)$ and satellite $\mathbf{R}_{s}(t)$, are specified by their vector components in an inertial Cartesian system of coordinates, such as ECI coordinates, by

$$
\begin{aligned}
& \mathbf{R}_{o}(t)=x_{o}^{i}(t) \mathbf{e}_{i} \\
& \mathbf{R}_{s}(t)=x_{s}^{i}(t) \mathbf{e}_{i}
\end{aligned}
$$

and $\mathbf{e}_{i}, i=1,2,3$ are the Cartesian basis vectors in the ECI (or other) inertial coodinates, $x_{o}^{i}(t)$ and $x_{s}^{i}(t), i=1,2,3$, are the time-dependent coordinates of receiver and satellite, respectively, and summation is implied when repeated indices occur, unless stated otherwise. The range from the satellite $s$ emmision event to the reception event at the receiver, in ECI inertial coordinates, is given implicitly by

$$
r_{s}=\left|\mathbf{R}_{o}(t)-\mathbf{R}_{s}\left(t-\frac{r_{s}}{c}\right)\right|
$$

The quantities $h_{i}\left(\mathbf{n}_{s}(t)\right)$ appearing in Eq. (16) are the components of the vector effective height of the receiving antenna projected on the ECI Cartesian coordinate basis vectors $\mathbf{e}_{i}$ and are given in terms of the spherical basis components in Eq. (12), by

$$
\mathbf{h}(\mathbf{n})=h_{\theta}(\theta, \phi) \mathbf{a}_{\theta}+h_{\phi}(\theta, \phi) \mathbf{a}_{\phi}=h_{1}(\mathbf{n}) \mathbf{e}_{1}+h_{2}(\mathbf{n}) \mathbf{e}_{2}+h_{3}(\mathbf{n}) \mathbf{e}_{3}
$$

where

$$
\begin{aligned}
& h_{1}(\mathbf{n})=h_{\theta}(\theta, \phi) \cos \theta \cos \phi-h_{\phi}(\theta, \phi) \sin \phi \\
& h_{2}(\mathbf{n})=h_{\theta}(\theta, \phi) \cos \theta \sin \phi+h_{\phi}(\theta, \phi) \cos \phi \\
& h_{3}(\mathbf{n})=-h_{\theta}(\theta, \phi) \sin \theta
\end{aligned}
$$

These components depend on the direction $(\theta, \phi)$ from which the radiation from satellite $s$ is incident on the receiving antenna. This direction can be specified by components of the unit vector $\mathbf{n}_{s}(t)$, defined in Eq. (17).

In Eq. (16), the matrix elements $D_{i k}(t)$ specify the attitude of the receiver platform at reception time $t$. The attitude is specified by giving the inner product of the unit vectors $\mathbf{a}_{i}, i=1,2,3$, of the receiver comoving coodinates with the ECI Cartesian coordinate basis vectors $\mathbf{e}_{i}$ :

$$
D_{i k}(t)=\mathbf{a}_{i}(t) \cdot \mathbf{e}_{i}
$$

where the vectors $\mathbf{a}_{i}(t)$ are time-dependent, since these vectors correspond to the moving body axes of the receiver. The receiver platform attitude matrix $D_{i k}(t)=D_{i k}(\alpha(t), \beta(t), \gamma(t))$ has three independent parameters. This attitude matrix can be conveniently represented in terms of three time-dependent Euler angles, $\alpha, \beta$, and $\gamma$, by [22]

$$
D_{i k}(t)=\left(\begin{array}{ccc}
\cos \beta \cos \alpha \cos \gamma-\sin \alpha \sin \gamma & \cos \beta \sin \alpha \cos \gamma+\cos \alpha \sin \gamma & -\sin \beta \cos \gamma \\
-\cos \beta \cos \alpha \sin \gamma-\sin \alpha \cos \gamma & -\cos \beta \sin \alpha \sin \gamma+\cos \alpha \cos \gamma & \sin \beta \sin \gamma \\
\sin \beta \cos \alpha & \sin \beta \sin \alpha & \cos \beta
\end{array}\right)
$$


The attitude matrix $D_{i k}(t)$ completely specifies the orientation of the basis vectors of the receiver platform, $\mathbf{a}_{i}$, in terms of the ECI basis vectors $\mathbf{e}_{i}$, see Figure 1.

In Eq. (16), the quantities $R_{1 k}\left(t-r_{s} / c\right)$ and $R_{2 k}\left(t-r_{s} / c\right)$ specify the satellite antenna polarization vectors, $\mathbf{u}$ and $\mathbf{v}$, at the time of transmission, $t-r_{s} / c$, with respect to the ECI Cartesian coordinate basis vectors $\mathbf{e}_{i}$ :

$$
\begin{aligned}
& \mathbf{u}\left(t-\frac{r_{s}}{c}\right)=\left(\mathbf{u} \cdot \mathbf{e}_{m}\right) \mathbf{e}_{m}=R_{1 m}\left(t-\frac{r_{s}}{c}\right) \mathbf{e}_{m} \\
& \mathbf{v}\left(t-\frac{r_{s}}{c}\right)=\left(\mathbf{v} \cdot \mathbf{e}_{m}\right) \mathbf{e}_{m}=R_{2 m}\left(t-\frac{r_{s}}{c}\right) \mathbf{e}_{m}
\end{aligned}
$$

In Eq. (16), the remaining quantities to be defined are

$$
k_{s}=N_{s} \frac{\omega}{c}=\frac{2 \pi N_{s} f}{c}
$$

where $N_{s}=N_{s 1}+j N_{s 2}$ is the effective index of refraction (real and imaginary) of the medium through which the electromagnetic wave travels, from satellite transmitter to the user's receiver. The frequency $f$ is the L-band (or other) carrier frequency.

The voltage in the receiving antenna due to signal from satellite $s$, given in Eq. (16), can be written as

$$
V_{s}(t)=v_{s} e^{-j \delta_{s}} E\left(\mathbf{n}_{s}\right) \frac{e^{-j k_{s} r_{s}}}{r_{s}} e^{j \omega t}
$$

where $v_{s}$ is a real amplitude and $\delta_{s}$ is a real phase angle, specified by (sums on repeated indices $i$ and $k$ ):

$$
\delta_{s}(t)=-\operatorname{Arg}\left\{h_{i}\left(\mathbf{n}_{\mathbf{s}}(t)\right) D_{i k}(t)\left[R_{1 k}^{(s)}\left(t-\frac{r_{s}(t)}{c}\right)-j R_{2 k}^{(s)}\left(t-\frac{r_{s}(t)}{c}\right)\right]\right\}
$$

Here, for real numbers $a$ and $\phi$, the function $\operatorname{Arg}\left(a e^{j \phi}\right)=\phi \bmod 2 \pi$.

The actual voltage in the receiving antenna is the real part of Eq. (16), which can be written as

$$
\operatorname{Re} V_{s}(t)=v_{s} \frac{E\left(\mathbf{n}_{s}\right)}{r_{s}} \cos \psi_{s}(t)
$$

where

$$
\psi_{s}(t)=k_{s} r_{s}(t)-\omega t+\delta_{s}(t)
$$

The phase of the voltage in the receiving antenna, $\psi_{s}(t)$ given in Eq. (34), should be compared with the phase of the scalar field, given in Eq. (2). The vector nature of the electromagnetic field leads to an additional phase shift $\delta_{s}(t)$ in Eq. (34). This phase shift is a function of the relative orientation of the satellite and receiver antenna. The single-antenna method of determining attitude exploits the dependence of the phase $\delta_{s}(t)$ on the relative orientation of the satellite and receiver antennas. Note that $\delta_{s}(t)$ in Eq. (32) depends on receiver antenna parameters $h_{i}\left(\mathbf{n}_{s}\right)$, which are defined in Eq. (22)-(24).

The model for the phase of the induced voltage in a receiving antenna, given in Eq.(32), is more accurate than the standard model in Eq. (2), because it takes into account the orientation of the receiving antenna in the electromagnetic field.

\section{IMPROVED CARRIER PHASE MEASUREMENT MODEL}

The satellite receiver has an electronic oscillator, whose phase is given by Eq. (值). The measured quantity is the phase difference between the local oscillator and the phase of the voltage in Eq. (34):

$$
\psi_{s}(t)-\psi_{\text {osc }}(t)=k_{s} r_{s}(t)+\omega \Delta t^{*}+\delta_{s}(t)+2 \pi m
$$

where $m$ is an integer, since the relative phase can be measured only up to integer multiples of $2 \pi$. For comparison purposes, Eq. (35) can be put into a notation similar to Eq. (7):

$$
\psi_{i s}(t)-\psi_{\text {osc }}(t)=k_{i s} r_{i s}(t)+\omega \Delta t^{*}+\delta_{i s}(t)+2 \pi m_{i s}
$$

where the subscript $i$ has been added to index the receiving antenna, and the subscript $s$ still refers to transmitting satellite $s$. Comparison of Eq. (36) with Eq. (34) and the previous definitions for $\delta_{s}(t)$ makes the meaning of the terms in Eq. (36) clear. 


\section{ATTITUDE DETERMINATION AND NAVIGATION}

\section{A. Multiple-Antenna Attitude Determination}

For the case of an attitude determination system that uses multiple antennas, Eq. (36) can now be used to form differences between two antennas and one satellite, such as is done in standard carrier phase tracking (see Eq.(8)):

$$
\begin{aligned}
\Delta \psi_{i s}(t)-\Delta \psi_{k s}(t) & =\left(k_{i s} r_{i s}(t)-k_{k s} r_{k s}(t)\right)+2 \pi\left(m_{i s}-m_{k s}\right)+\delta_{i s}(t)-\delta_{k s}(t) \\
& \approx k\left\{\left(\mathbf{r}_{k}-\mathbf{r}_{i}\right) \cdot \frac{\mathbf{R}_{j}}{\left|\mathbf{R}_{j}\right|}\right\}+2 \pi\left(m_{i s}-m_{k s}\right)+\delta_{i s}(t)-\delta_{k s}(t)
\end{aligned}
$$

where $\mathbf{r}_{i}$ are the positions of the receiving antennas at reception time $t$. The measured phase, as given by Eq. (38), contains an additional difference of phase shifts, $\delta_{i s}(t)-\delta_{k s}(t)$, which depends on two attitude matrices, $D_{i s}$ and $D_{k s}$, one for each of the two antennas mounted on the user platform. Compare Eq.(38) with Eq.(9). When the two antennas are identical, and they are mounted on a rigid platform, and they are aligned, then these two matrices are the same. The difference of phase shifts $\delta_{i s}(t)-\delta_{k s}(t)$ is essentially zero. Alternatively, if these conditions are not satisfied, then Eq.(38) provides the generalization of Eq.(9), and includes the effects of flexing of the platform and misalignment of the antennas.

\section{B. Single-Antenna Attitude Determination}

The orientation dependence of the phase shift $\delta_{i}(t)$ in Eq. (32) can be exploited to permit determination of attitude (by determining $\alpha, \beta$, and $\gamma$ ) from carrier phase measurements made by a single antenna. Consider a receiver whose antenna has a known orientationa and position at some initial time $t_{o}$. The phase change of the oscillator in the receiver, since the initial time $t_{o}$ can be represented by

$$
\Delta \psi_{\text {osc }}(t)=-\omega\left(t^{*}-t_{o}\right)
$$

where $t$ is the GPS time (or coordinate time in the ECI (or other) frame of reference) that is associated with a time $t^{*}$ on the clock that counts oscillator cycles 16. The oscillator in the user's receiver is assumed to be imperfect. The imperfection of the oscillator can be represented by the fact that the oscillator does not keep time according to ECI coordinate time. The oscillator will then have a running phase given by

$$
\psi_{o s c}(t)=-\omega t^{*}
$$

where the time scale $t^{*}$ is not the same as the ECI coordinate time $t$. I assume that at the initial time $t_{o}$ the oscillator has the correct time $t^{*}=t_{o}$. If we counted the cycles of the oscillator since the initial time $t_{o}$, and multiplied by the period of the oscillator, $2 \pi / \omega$, then the elapsed time, according to the oscillator is $t^{*}-t_{o}$. The difference between the real ECI coordinate time elapsed, $t-t_{o}$, and the elapsed time as computed by counting oscillator cycles, $t^{*}-t_{o}$, is given by $\left(t-t_{o}\right)-\left(t^{*}-t_{o}\right)=t-t^{*} \equiv \Delta t^{*}$, where $\Delta t^{*}$ is the oscillator clock bias that has accrued since the initial time. So the time kept by the oscillator, $t^{*}$ and the coordinate time, $t$, are related by

$$
t^{*}=t-\Delta t^{*}
$$

Using Eq. (41) in Eq. (5) gives the oscillator phase change since the initial time $t_{o}$ in terms of ECI coordinate time and the oscillator clock bias

$$
\Delta \psi_{\text {osc }}(t)=-\omega\left(t-t_{o}-\Delta t^{*}\right)
$$

The phase of the voltage (due to satellite $s$ ) in the receiver antenna is given by Eq.(34). Since the initial time $t_{o}$, the phase of the voltage in the antenna changed by

$$
\Delta \psi_{s}(t) \equiv \psi_{s}(t)-\psi_{s}\left(t_{o}\right)=k_{s}\left(r_{s}(t)-r_{s}\left(t_{o}\right)\right)-\omega\left(t-t_{o}\right)+\delta_{s}(t)-\delta_{s}\left(t_{o}\right)
$$

By taking the difference of the phase changes that have accrued on the receiver's oscillator (see Eq. (42)) and the carrier phase from satellite $s$ (see Eq. (43)), I get the single antenna carrier phase-change equation

$$
\Delta \Psi_{s}(t) \equiv \Delta \psi_{s}(t)-\Delta \psi_{o s c}(t)=k_{s}\left(r_{s}(t)-r_{s}\left(t_{o}\right)\right)-\omega \Delta t^{*}+\delta_{s}(t)-\delta_{s}\left(t_{o}\right)
$$


Equation (44) gives the difference of the accrued phase changes in the oscillator and in the voltage of the receiver (due to signal of satellite $s$ ). This is the quantity that is measured by satellite tracking receivers and is known as a measurement of carrier phase or accumulated delta-range. Equation (44), together with the above definitions, defines an algorithm for single-antenna determination of position, time, and attitude by satellite navigation.

The measured phase change given by Eq. (44) depends on seven unknown parameters and several assumed-known parameters:

$$
\Delta \Psi_{s}\left(x_{o}^{k}, \Delta t^{*}, \alpha, \beta, \gamma ; x_{s}^{k}, R_{1 k}, R_{2 k}, \alpha_{o}, \beta_{o} \gamma_{o}\right)
$$

The unknown parameters at time $t$ are the receiver antenna position, $x_{o}^{k}, k=1,2,3$, receiver oscillator phase correction $\omega \Delta t^{*}$ (or equivalently, the receiver clock bias $\Delta t^{*}$ ), and the three Euler angles, $\alpha, \beta$, $\gamma$, which define the attitude matrix of the receiver antenna. The parameters that are assumed known functions of time are the satellite position coordinates or satellite ephemeris, $x_{s}^{k}, k=1,2,3$, and the satellite attitude parameters $R_{1 k}, R_{2 k}, k=1,2,3$. I will collectively refer to $x_{s}^{k}, R_{1 k}$ and $R_{2 k}$, as the satellite enhanced ephemeris. In addition, the receiver attitude at the initial time $t_{o}$ must be known through, for example, the Euler angles $\alpha_{o}, \beta_{o}$, and $\gamma_{o}$.

There are several modes in which a user may exploit the general single antenna attitude determination algorithm described above. The choice of mode depends on other systems that are available to the user, and what information the user requires. Perhaps the most basic assumption is that the user has no additional systems for navigation. In this case, the user receiver determines the needed parameters at the initial time $t_{o}$, as well as the satellite enhanced ephemeris, and then tracks the carrier phase continuously of seven satellites. At a given time $t$, the seven equations in Eq. (44), for $s=1, \cdots, 7$, are a closed system of equations that can be solved for the seven parameters of receiver position, $x_{o}^{k}, k=1,2,3$, receiver clock bias $\Delta t^{*}$, and the complete three-axis attitude given by the three Euler angles $\alpha, \beta$, and $\gamma$. The requirement that the user's receiver track seven satellites is not as severe as it may seem, because, for example, in the case of GPS, there are plans to provide additional satellites to the number that are currently operational.

Another mode of navigation is to carry an oscillator that is sufficiently stable over the intended time interval of navigation. For example, in the case of a UAV, the time of flight may be 10 minutes. An oscillator accurate to one part in $10^{9}$ would maintain a phase within

$$
\omega \Delta t^{*}=2 \pi\left(1200 \mathrm{~s}^{-1}\right)\left((600 s)\left(10^{-9}\right)\right)=0.0045 \text { radian } \approx 0.26^{\circ}
$$

If the oscillator is accurate enough, then the parameter $\Delta^{*} t$ does not have to be determined, and it is sufficient to track six satellites, to compute the three position coordinates and the three Euler angles of attitude.

Still another mode of navigation is to provide the user with compass heading information. The ability to determine three axis attitude allows the extraction of the compass heading associated with a particular axis of the receiver platform. This requires a trivial transformation from ECI coordinates and time to topocentric coordinates.

\section{SUMMARY}

Current technology of attitude determination by satellite navigation requires the use of multiple, well-separated antennas. In this paper, I have described the theory and the algorithm for determining the position, time and three angles of attitude (antenna orientation), when tracking the carrier phase of seven satellites using a single antenna. The method is based on the phase change induced in the open circuit voltage in an antenna due to the signal of a satellite that is transmitting from a known location and having a known orientation. The dependence of the phase shift on receiving antenna orientation is given in Eq. (32).

The attitude determination algorithm described here can be applied by current GPS receivers with essentially a software addition, since these receivers routinely track the carrier phase (or delta range) of the satellites. Furthermore, the orientation of the GPS antennas is known quite accurately because the satellite main beam is pointed toward the center of the Earth 12].

If a higher accuracy of attitude is required, the satellite broadcast ephemeris can be enhanced to include precise information on the satellite antenna orientations. This enhanced ephemeris could be implemented in a future version of the GPS, or some other satellite system. Such an architecture would make the satellite system capable of providing a user with time, position, and orientation.

For user applications, a single-antenna (rather than multiple-antenna ) attitude determination system is preferable because it allows miniaturization of the receiver system and is potentially less expensive to manufacture and to install. An example where single-antenna attitude determination is preferable is in micro air vehicles (MAV), nano satellites [13] and in general aviation aircraft. Currently, the U.S. fleet of aircraft is transitioning from groundbased navigation methods to satellite-based (mostly GPS) navigation. Attitude information is needed by a pilot 
whenever aircraft are flying by reference to instruments, such as in instrument meteorological conditions (IMC). Many general-aviation aircraft already have single-antenna GPS receivers. Therefore, it may be possible to extract attitude information, using the single-antenna attitude determination method presented here, from GPS carrier phase tracking data in already-installed GPS receivers, with only a software change and addition of an attitude output channel.

Information on position, time and attitude of a platform is equivalent to knowing the compass heading. Consequently, another application of attitude determination is to replace the wet compass. Satellite navigation receivers are being miniaturized and put into a wrist watch [15]. The potential for miniaturizing the user receiver by using the single-antenna attitude determination method may lead to production of a satellite-based compass that can be carried by a person in a watch-sized instrument.

Finally, the algorithm presented here can be applied to GPS signal simulators, to simulate a real signal seen by a satellite receiver that is rotating as well as translating.

[1] Global Positioning System: Theory And Applications, vol. I and II, B. W. Parkinson and J. J. Spilker, eds. Progress in Astronautics and Aeronautics, vol. 163 and 164, American Institute of Aeronautics and Astronautics, Washington, DC, 1996.

[2] B. Hofmann-Wellenhof, H. Lichtenegger, and J. Collins, "GPS Theory and Practice", Third Revised Edition, Springer-Verlag, New York (1994).

[3] Kaplan, E. D. (1996). In Understanding GPS: Principles and Applications, (Mobile Communications Series, Artech House, Boston).

[4] For an overview, see for example, C. E. Cohen, "Attitude Determination," Chapter 19 in Global Positioning System: Theory And Applications, vol. II, B. W. Parkinson and J. J. Spilker, eds. Progress in Astronautics and Aeronautics, vol. 163 and 164, American Institute of Aeronautics and Astronautics, Washington, DC, 1996.

[5] A. Krall and T. B. Bahder, "Orientation and Velocity Effects in the Global Positioning System: Single-Antenna Attitude Determination", manuscript accepted for publication in J. Appl. Phys.

[6] A. Krall and T. B. Bahder, "Single Antenna Method for Attitude Determination using the Global Positioning", manuscript submitted to IEEE Transactions on Aerospace Systems, August 2000.

[7] The dependence of the voltage on the orientation of the receiving and transmitting antennas leads to two well-known effects: "movement of the phase center" of the receiving antenna, and "phase wind-up" or an additional effective wavelength in range for each complete rotation of receiving antenna about the line of site to the satellite.

[8] G. Sinclair, "Transmission and Reception of Elliptically Polarized Waves", Proc. I.R.E. 38, 148-151 (1950).

[9] R. E. Collin and F. J. Zucker, "Antenna Theory, Part I", McGraw-Hill Book Company, New York (1969).

[10] G. H. Price, "On the Relationship Between the Transmitting and Receiving Properties of an Antenna", IEEE Trans. Ant. Prop. AP-34, 1366-1368 (1986).

[11] C. A. Balanis, Antenna Theory, Second Edition, J. Wiley and Sons, Inc., New York (1997).

[12] For the case of GPS satellites, the attitude is controlled quite precisely: the nadir pointing accuracy is $0.5 \mathrm{deg} / \mathrm{sec}(5 \sigma)$ and the angular rates must be less than $0.5 \mathrm{deg} / \mathrm{sec}$ or $5 \times 10^{-5} \mathrm{deg}$ in $1 \mathrm{~ms}(3 \sigma)$; the yaw attitude control accuracy is better than $3.0 \mathrm{deg}(3 \sigma)$ with respect to the sun. See the document, "System Segment Specification for the GPS Production Space Segment, Vol. 5 Attitude and Velocity Control Subsystem, NAVSTAR GPS Joint Program Office, 11 Feb. 1988.

[13] R. F. Turner, "Small spacecraft missions-the US scene", Proceedings of the Institution of Mechanical Engineers ( London), 213, 213, (1999).

[14] B. Nordwall, "Micro Air Vehicles Hold Great Promise, Challenges," Aviation Week \& Space Technology, April 14, pp. 67, 1997.

[15] Pierre-Andre Farine, "Watch your GPS", GPS World, pp. 24, April 2001.

[16] There need not be an actual clock in the receiver to count oscillator cycles. The time $t^{*}$ simply marks the phase of the oscillator, so the concept of a clock is here a theoretical construct.)

[17] Here, I neglect the small effect of relativistic aberration, i.e., change of apparent propagation direction when the radiation field is observed from a frame of reference that is moving (the receiver's comoving frame) with respect to the source of the field.

[18] V. H. Rumsey, "Part I-Transmission Between Elliptically Polarized Antennas", Proc. IRE 39, 535-540 (1961).

[19] G. A. Deschamps, "Part II-Geometrical Representation of the Polarization of a Plane Electromagnetic Wave", Proc. IRE 39, 540-544 (1961).

[20] M. L. Kales, "Part III-Elliptically Polarized Waves and Antennas", Proc. IRE 39, 544-549 (1961).

[21] J. I. Bohnert, "Part IIV-Measurements on Elliptically Polarized Antennas", Proc. IRE 39, 549-552 (1961). 
[22] J. Mathews and R. L. Walker, p. 404 in Methermatical Methods of Physics, 2nd Edition, W. A. Benjamin, Inc. Menlo Park, California, U.S.A. (1970).

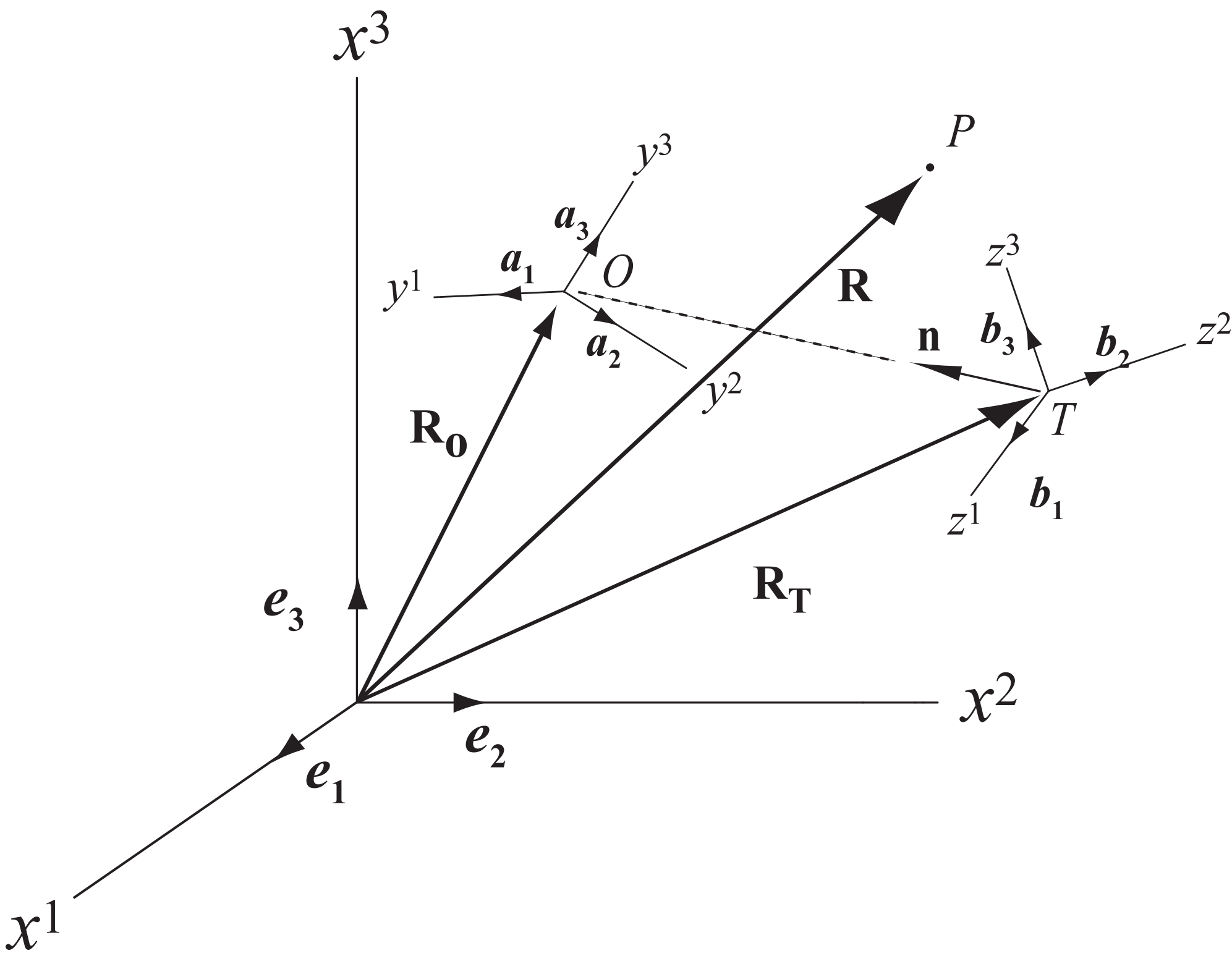

FIG. 1. The Earth-centered coordinates $x^{i}$ with basis vectors $\mathbf{e}_{i}, i=1,2,3$, is shown together with the comoving system of coordinates $y^{i}$ centered at the receiver (point $O$ ) and the satellite comoving system of coordinates $z^{i}$ centered at point $T$. The unit vector $\mathbf{n}$ points from the emission event at the satellite at time $t-r_{s} / c$ to the reception event at the receiver at time $t$. 